\title{
Construcción de una escala de evaluación de la Inteligencia emocional para niños de primer ciclo básico \\ Catalina Moore Infante ${ }^{1}$ \\ J.Mauricio Soto Retamal ${ }^{2}$
}

\section{Resumen}

El objetivo del presente trabajo es construir una Escala para evaluar la Inteligencia Emocional en niños de primer ciclo básico de la ciudad de Concepción. Esto responde a la ausencia de otros instrumentos estadisticamente válidos y confiables para este constructo, y pretende ser un aporte para potenciar el desarrollo emocional de los niños, en el marco de los Objetivos Transversales de la Reforma Educacional en marcha.

La construcción de los items de la Escala, compuesta por cinco dimensiones, fue realizada a través de un proceso convergente a partir de la revisión teórica y de la experiencia personal de los profesores de aula. El instrumento fue aplicado a una muestra probabilistica de 321 niños de ambos sexos (162 varones y 159 mujeres), de dos niveles socioeconómicos (bajo y medio alto).

Los resultados del análisis estadístico muestran una confiabilidad de la Escala Total de 0.94, medida a través del coeficiente de correlación de Kuder-Richardson (KR-20). Asimismo, se encontró que todos los reactivos presentaron un alto poder de discriminación.

El análisis factorial muestra una alta concentración de los itemes en torno a una única dimensión general, lo que no permitiria confirmar la existencia de las cinco dimensiones descritas habitualmente en la literatura.

El instrumento se encuentra actualmente en su última etapa de estandarización, para la provincia de Concepción.

Palabras Claves: Inteligencia Emocional, Dimensiones, Evaluación, Edad Escolar

\section{Abstract}

The purpose of the present study is to construct a Scale to evaluate Emotional Intelligence in scholar children betwen first to fourth grade in the city of Concepcion (Chile). This is motivated by the absence of other instruments statistically valid and reliable to measure this concept, and pretends to be a contribution to potenciate the child emotional development, in the context of the Transversal Objectives of the Chilean Educational Reform.

The construction of the items of the Scale formed by five dimensions, was made by a convergent process through the theoretical review and the personal experience of teachers. This pilot instrument was applied to an intencional sample of 321 chidren of both sexes (162 boys and 159 girls), from two different socioeconomic levels (low and high). 
The results of the statistical analysis show a reliability of the Total Scale of 0.94 , measured by the Kuder Richardson correlation coeficient (KR-20). Also, it was found that all the items had high discrimination power.

The factor analysis shows a high concentration of the items around one general dimension; this does not permit to confirm the five dimensions previously established.

The instrument is undergoing the last part of the process of standarization, for the province of Concepción.

Key words: Emotional Intelligence, Dimensions, Measurement, Scholar Age.

\section{Definición del concepto ysusdimensiones}

El concepto de Inteligencia Emocional despierta cada día mayor interés tanto en el terreno psicoeducacional como empresarial, pues se ha constatado que la inteligencia entendida sólo en términos de habilidades cognitivas, no da cuenta del éxito y capacidad de adaptación del ser humano (Goleman, 1995 en Young, 1998).

Salovey y Mayer fueron los primeros en acuñar y definir este término en el año 1990, refiriéndose a él como un "subconjunto de la inteligencia social que comprende la capacidad de controlar los sentimientos y emociones propias asi como las de los demás, discriminar entre ellas y utilizar esta información para guiar nuestro pensamiento y nuestras acciones" (Shapiro, 1997, pag. 27).

Goleman (1996) por su parte, enumera las siguientes habilidades que compondrian la Inteligencia Emocional: capacidad de automotivarse, persistencia, autocontrol emocional, capacidad para demorar la gratificación, capacidad de empatía y capacidad de manejar las relaciones interpersonales de una manera fluida.

En la presente investigación, se elaboró una definición de Inteligencia Emocional a partir de lo postulado por los autores antes mencionados, la que hace referencia a la "capacidad del niño para enfrentar con tranquilidad, optimismo y perseverancia las dificultades, establecer relaciones interpersonales positivas, comprender los sentimientos y necesidades propias y de los demás, y

\section{usar esta información para guiar y controlar la conducta".}

Entre otros elementos, esta definición destaca que la información derivada de las experiencias emocionales ayuda a solucionar eficazmente los problemas y a lograr una mejor adaptación de la conducta, en la medida que incluye por una parte, una evaluación de elementos tanto internos como externos, como también aspectos de carácter afectivo y cognitivo.

Goleman (1996), uno de los autores que más ha investigado en el campo de la inteligencia emocional, fundamenta estos planteamientos en datos empíricos de la realidad social. Entre otros, en el estudio de los efectos de la agresividad, la impulsividad, la ansiedad y el autocontrol de la conducta humana; la reflexión sobre casos de la vida real en los que las emociones originan, orientan y dirigen la conducta inteligente; $y$ en el análisis de otros enfoques de la inteligencia que destacan también la dimensión emocional en el funcionamiento intelectual. Los resultados de sus investigaciones le hacen concluir que la inteligencia académica no nos prepara para las vicisitudes que nos depara la vida.

Aún cuando tener un alto cociente intelectual no garantiza el nivel de prosperidad, prestigio o felicidad en la vida, nuestra educación formal y las demandas culturales siguen poniendo mayor énfasis en las habilidades cognitivas, ignorando muchas veces las habilidades emocionales que afectan a nuestra capacidad de autorregularnos en el comportamiento diario. Para Goleman (1996), la formación de los sentimientos debe tener un lugar rele- 
vante en el currículum académico, como lo tienen las matemáticas y el lenguaje. Considera que la escuela debe ejercer una función más directa en la formación de las actitudes, de los sentimientos y del autoconcepto de los niños. Al respecto, existe amplio acuerdo entre los especialistas, en que estas habilidades son altamente dependientes del medio ambiente, y que por lo tanto, son factibles de desarrollar.

Esta interpretación de la inteligencia emocional de Goleman, es visiblemente apoyada por otros autores que han estudiado la dimensión cognitiva del ser humano. En esta linea podemos mencionar a Feuerstein y sus colaboradores, a Gardner y a Stenberg (Henríquez, Saez, Valenzuela y Villena, 1998). En concreto, Gardner puntualiza la importancia y la necesidad de comprender las motivaciones y emociones individuales, no sólo en el ámbito personal, sino también en la interacción con los otros. Valora en la misma medida que las habilidades cognoscitivas, estos aspectos personales, a los que atribuye el rol de nuevas inteligencias, denominadas Interpersonal (conocimiento de los otros) e Intrapersonal (conocimiento del yo). La educación formal, para él, debe estimular al máximo las inteligencias personales, porque son las que realmente educan a los líderes sociales y ayudan a reconocer los talentosy limitaciones personales, aspectos esenciales en una sociedad democrática (Gardner, 1987).

Hoy en dia, existe consenso entre los distintos autores acerca de la existencia de cinco dimensiones fundamentales que formarian parte de este constructo. De acuerdo a lo postulado por Salovey y Mayer y de posteriores especificaciones hechas por Goleman (Goleman, 1998; Molero,1998) éstas pueden ser conceptualizadas como:

a) Autoconciencia: Se refiere al conocimiento de uno mismo, de nuestros propios sentimientos, saber quiénes somos. El reconocer nuestros sentimientos nos da un mayor control sobre nuestras vidas $y$, por el contrario, la incapacidad para reconocerlos nos deja a su merced.

b) Autorregulación: Se refiere al manejo de los propios estados de ánimo, impulsos y emocio- nes. Significa que el sujeto es capaz de mantener bajo control sus emociones perturbadoras tanto en momentos de decaimiento como de entusiasmo, evitando los sentimientos prolongados de ansiedad, irritabilidad, etc.

c) Automotivación: Se refiere a las tendencias emocionales que guían o facilitan el cumplimiento de las metas establecidas. La capacidad de automotivarse implica regular las emociones al servicio de una meta, e incluye la persistencia y el entusiasmo ante los contratiempos.

d) Empatía: Es la habilidad relacional más importante. Implica tener conciencia de los sentimientos, necesidades y preocupaciones de los otros y es la antesala del altruismo.

e) Relaciones Interpersonales: Se refiere a la habilidad para la competencia social. Esto implica la capacidad para establecer un encuentro con el otro, prosperar en las relaciones intimas, persuadir e influir y manejar las emociones de los demás.

Mayery Salovey (1993, en Goleman, 1996), plantean que la evaluación de la inteligencia emocional tiene sentido, porque se trata de una habilidad que facilita la adaptación social, la salud mental y la productividad académica.

Si consideramos el creciente aumento de problemas en elárea de la salud mental infantil, se hace muy necesario orientar los esfuerzos a desarrollar las habilidades emocionales de los niños como una forma de prevenir trastornos posteriores. Para ello se requiere contar con algún instrumento que permita evaluar las competencias antes mencionadas.

El presente estudio pretende llenar el vacio existente en este campo, dada la ausencia de tests estadísticamente válidos y confiables que evalúen la Inteligencia Emocional (Young, 1998).

\section{Metodología}

La definición antes señalada y las cinco dimensiones que la componen, fueron el marco de referencia para la construcción de los ítemes de la Esca- 
la. A partir de esta información, se confeccionó una Escala con cinco dimensiones, cuyos indicadores fueron elaborados a través de un doble proceso: deductivo e inductivo. El procedimiento deductivo consistió en la elaboración de ítemes a partir de la revisión teórica del constructo. El inductivo, consistió en la consulta a profesores acerca de las conductas que ellos consideraban representativas de los niños con alta y baja inteligencia emocional, toman- los cuales debía evaluar a 3 o 4 alumnos de su curso seleccionados previamente al azar por los investigadores.

A partir de los resultados obtenidos del análisis de la aplicación a la muestra piloto, se realizaron las siguientes modificaciones al instrumento:

1. Agregar 6 nuevos reactivos con el objeto de reemplazar aquellos que en la aplicación pilo-

\begin{tabular}{|lccccc|}
\hline \multicolumn{7}{c|}{ Tabla 1 } \\
\multicolumn{7}{c}{ Distribución de la muestra definitiva según NSE, Sexo y Curso. } \\
Curso & NSE Medio Alto (N=167) & \multicolumn{2}{c|}{ NSE Bajo (N=154) } & Total \\
$1^{\circ}$ & Mujer & Hombre & Mujer & Hombre & \\
$2^{\circ}$ & 24 & 24 & 21 & 24 & $\mathbf{B}$ \\
$3^{\circ}$ & 21 & 26 & 18 & 15 & $\mathbf{8}$ \\
$4^{\circ}$ & 18 & 18 & 18 & 18 & $\mathbf{7 2}$ \\
Total & 18 & 18 & 21 & 19 & $\mathbf{7}$ \\
& $\mathbf{8 1}$ & $\mathbf{6}$ & $\mathbf{B}$ & $\mathbf{6}$ & $\mathbf{3 2}$ \\
\hline
\end{tabular}

do como referencia la definición de Inteligencia Emocional anteriormente descrita.

Esta primera etapa dio como resultado alrededor de 65 itemes. Estos cuales fueron sometidos a juicio de expertos, aceptándose sólo aquellos reactivos en los que había un $80 \%$ de acuerdo entre los 5 evaluadores. La tarea de los jueces comprendia tres objetivos: señalar si cada item se desprendía o no de la definición entregada, si permitía discriminar entre alta y baja inteligencia emocional y a cuál de las cinco dimensiones pertenecería. Finalmente, se aceptaron 30 items, 6 para cada una de las dimensiones.

Para determinar las caracteristicas técnicas de la Escala, se realizó una aplicación piloto a una muestra intencionada (por accesibilidad) de 41 alumnos de primer ciclo básico, de ambos sexos(19 mujeres y 22 hombre) y de dos niveles socioeconómicos (bajo y medio alto) de la ciudad de Concepción. El instrumento, de carácter dicotómico, debía ser respondido por el profesor. Se utilizó un grupo de 16 profesores, cada uno de to mostraron una baja correlación ítem-test.

2. Transformar la modalidad de respuesta dicotómica de la Escala, por una con cuatro posibilidades: nunca/ a veces/generalmente/ siempre. Esto por dos motivos principales: sugerencia de los profesores y dificultad para realizar un análisis factorial confiable con una escala de ese tipo.

La Escala definitiva quedó constituída por 30 itemes, cinco para cada dimensión, y fue aplicada a una muestra probabilistica de 321 niños de primer ciclo básico, de ambos sexos y dos niveles socioeconómicos (ver tabla $\mathrm{N}^{\circ} 1$ ).

\section{Resultados}

\section{a) Análisis de la Confiabilidad del Instru- mento Definitivo.}

Para estimar la confiabilidad del instrumento, tanto respecto a la Escala Total como a las cinco dimensiones

(1-Autoconocimiento, 


\section{Tabla2}

Confiabilidad Total y de cada Dimensión

\begin{tabular}{lcccccc}
\hline Dimensión & 1 & 2 & 3 & 4 & 5 & Escala Total \\
Kr 20 & 0,79 & 0,71 & 0.9 & 0,8 & 0,85 & $\mathbf{0 , 9 4}$
\end{tabular}

2-Autorregulación, 3-Motivación, 4-Empatíay 5-Relaciones Interpersonales), se empleó el Coeficiente de Confiabilidad de Kuder-Richardson (ver Tabla $\mathrm{N}^{\circ} 2$ ).

Los resultados de la Tabla 2 muestran que la confiabilidad de la Escala Total resultó ser muy adecuada, obteniéndose un coeficiente de 0.94 . Las cinco subescalas fueron también confiables, fluctuando sus coeficientes de la siguiente manera: 0.9 para motivación, 0.85 para relaciones interpersonales, 0.8 para empatia, 0.79 para autoconocimientoy 0.71 para autorregulación.

\section{b) Análisis deltemes}

A continuación en la Tabla 3 se presentan los resultados del análisis de las correlaciones Item-Test (r p.b.).

La consistencia interna de la Escala, medida por la vía de correlacionar cada ítem con el puntaje total, fue muy adecuada. Como se puede apreciar en la Tabla 3, todos sus reactivos se correlacionan positiva y significativamente con la Escala Total con un $\mathrm{p}<0.001$.

En la Tabla 4 se presentan los análisis de las correlaciones item-dimensión (r p.b.). En relación a la consistencia interna de las subescalas, ésta resultó alta, puesto que la totalidad de los ítemes, salvo el ítem 8 de la dimensión autorregulación, tienen una correlación positiva y significativa con las escalas respectivas $(\mathrm{p}<0.001)$. Esto es altamente coincidente con los resultados obtenidos en las correlaciones item-test y con los indices de confiabilidad de las escalas, siendo la dimensión autorregulación la que presenta una menor confiabilidad (0.71).

\begin{tabular}{|c|c|}
\hline Con & $\begin{array}{l}\text { bla3: } \\
\text { m-Test Corregido }\end{array}$ \\
\hline Item & Correlación \\
\hline 1 & 0,612 \\
\hline 2 & 0,679 \\
\hline 3 & 0,409 \\
\hline 4 & 0,554 \\
\hline 5 & 0,542 \\
\hline 6 & 0,532 \\
\hline 7 & 0,292 \\
\hline 8 & 0,474 \\
\hline 9 & 0,356 \\
\hline 10 & 0,319 \\
\hline 11 & 0,374 \\
\hline 12 & 0,421 \\
\hline 13 & 0,685 \\
\hline 14 & 0,667 \\
\hline 15 & 0,711 \\
\hline 16 & 0,655 \\
\hline 17 & 0,638 \\
\hline 18 & 0,649 \\
\hline 19 & 0,664 \\
\hline 20 & 0,483 \\
\hline 21 & 0,427 \\
\hline 22 & 0,617 \\
\hline 23 & 0,641 \\
\hline 24 & 0,693 \\
\hline 25 & 0,597 \\
\hline 26 & 0,686 \\
\hline 27 & 0,640 \\
\hline 28 & 0,657 \\
\hline 29 & 0,626 \\
\hline \multirow[t]{2}{*}{30} & 0,625 \\
\hline & $\begin{array}{c}\text { Todas las correlaciones } \\
\text { significativas } \\
p>0,001\end{array}$ \\
\hline
\end{tabular}




\begin{tabular}{|c|c|c|c|c|c|c|c|c|c|}
\hline \multicolumn{10}{|c|}{$\begin{array}{c}\text { Tabla N } 4 \text { : } \\
\text { Correlaciones Item-Dimensión Corregido }\end{array}$} \\
\hline \multirow{3}{*}{\multicolumn{3}{|c|}{$\begin{array}{l}\text { Correlación } \\
\text { Item-Dimensión1 } \\
\text { Corregido }\end{array}$}} & \multirow{3}{*}{$\begin{array}{l}\text { Correlación } \\
\text { Item-Dimensión } \\
\text { Corregido }\end{array}$} & \multirow{3}{*}{\multicolumn{2}{|c|}{$\begin{array}{c}\text { Correlación } \\
\text { Item-Dimensión3 } \\
\text { Corregido }\end{array}$}} & \multirow{3}{*}{\multicolumn{2}{|c|}{$\begin{array}{c}\text { Correlación } \\
\text { Item-Dimensión4 } \\
\text { Corregido }\end{array}$}} & \multirow{3}{*}{\multicolumn{2}{|c|}{$\begin{array}{c}\text { Correlación } \\
\text {-Dimensión5 } \\
\text { Corregido }\end{array}$}} \\
\hline & & & & & & & & & \\
\hline & & & & & & & & & \\
\hline P1 & 0,526 & P7 & 0,465 & P13 & 0,767 & P19 & 0,662 & P25 & 0,522 \\
\hline P2 & 0,591 & P8 & $\left.0,109{ }^{*}\right)$ & P14 & 0,740 & $\mathrm{P} 20$ & 0,436 & P26 & 0,6789 \\
\hline P3 & 0,469 & P9 & 0,545 & P15 & 0,772 & $\mathrm{P} 21$ & 0,455 & P27 & 0,5879 \\
\hline P4 & 0,674 & P10 & 0,512 & P16 & 0,763 & $\mathrm{P} 22$ & 0,533 & P28 & 0,6838 \\
\hline P5 & 0,641 & P11 & 0,640 & P17 & 0,677 & P23 & 0,607 & P29 & 0,6815 \\
\hline P6 & 0,393 & P12 & 0,511 & P18 & 0,702 & P24 & 0,632 & P30 & 0,6151 \\
\hline Alfa & 0,79 & Alfa & 0,71 & Alfa & 0,9 & Alfa & 0,8 & Alfa & 0,85 \\
\hline
\end{tabular}

\section{c) AnálisisFactorial}

Se realizó un análisis factorial (ver tablas 5 y 6 ) que mostró la existencia de una gran dimensión que concentra la mayoría de los itemes de la Escala (21 de 30), y una posible segunda dimensión compuesta por 4 itemes, que sin embargo no corresponde a ninguna de las dimensiones previamente definidas. Esto significa que no se puede confirmar la existencia de las cinco dimensiones planteadas en la literatura, sino la presencia de una gran dimensión compuesta por competencias emocionales de las distintasáreas.

\begin{tabular}{|cccc|}
\hline \multicolumn{5}{|c|}{ Tabla5 } \\
Análisis Factorial según Método deExtracción \\
deComponentes Principales \\
Autovalores & Varianza & Varianza \\
Componente & Total & explicada & $\begin{array}{r}\% \\
\text { acumulado }\end{array}$ \\
& & & 37,561 \\
1 & 11,268 & 37,561 & 48,563 \\
2 & 3,300 & 11,001 & 54,119 \\
3 & 1,667 & 5,556 & 59,07 \\
4 & 1,485 & 4,952 & 62,743 \\
5 & 1,102 & 3,673 & 65,906 \\
6 & 0,949 & 3,162 & 68,62 \\
7 & 0,814 & 2,714 & 71,221 \\
8 & 0,78 & 2,601 & 73,555 \\
9 & 0,7 & 2,333 & 75,715 \\
10 & 0,648 & 2,16 & \\
\hline
\end{tabular}

\section{d) Análisis Complementarios}

Como una manera de confirmar la necesidad de obtener normas para los distintos grupos de la población, se realizó un análisis de las diferencias de puntajes según las características que definen a la muestra.

\section{Diferenciaspor nivel socioeconómico}

Como se puede observar en la tabla $\mathrm{N}^{\circ} 7$, existen diferencias significativas en las puntuaciones de la escala total $(p<0.001)$ entre ambos niveles socioeconómicos, favoreciendo al grupo de sector medio-alto.

\begin{tabular}{|c|c|c|c|c|c|}
\hline \multicolumn{6}{|c|}{$\begin{array}{c}{\text { Tabla } \mathrm{N}^{\circ} 7}_{\text {Comparación de Puntajes Totales según NSE. }}\end{array}$} \\
\hline NSE & $\mathrm{n}$ & Media & $\begin{array}{c}\text { Desviación } \\
\text { Estandar }\end{array}$ & Min & Max \\
\hline Medio Alto & 167 & 64,970 & 13,346 & 25 & 90 \\
\hline Bajo & 154 & 59,344 & 15,952 & 14 & 90 \\
\hline \multirow[t]{2}{*}{ Total } & 321 & 62,271 & 14,899 & 14 & 90 \\
\hline & $=11$ & $p<0, c$ & & & \\
\hline
\end{tabular}




\section{Tabla $N^{\circ} 6$}

Matriz Factorial para los Primeros Cinco Componentes

\begin{tabular}{|c|c|c|c|c|c|}
\hline Item & Factor 1 & Factor 2 & Factor 3 & Factor 4 & Factor 5 \\
\hline 1 & 0.642 & 0.151 & 0.211 & 0.121 & 0.266 \\
\hline 2 & 0.715 & 0.07889 & 0.01553 & 0.04216 & 0.23 \\
\hline 3 & 0.461 & 0.615 & 0.15 & 0.259 & 0.09797 \\
\hline 4 & 0.586 & 0.329 & 0.291 & 0.232 & 0.349 \\
\hline 5 & 0.585 & 0.505 & 0.247 & 0.208 & 0.263 \\
\hline 6 & 0.565 & 0.349 & 0.01298 & 0.147 & 0.382 \\
\hline 7 & 0.304 & 0.484 & 0.005644 & 0.502 & 0.297 \\
\hline 8 & 0.51 & 0.152 & 0.471 & 0.275 & 0.0555 \\
\hline 9 & 0.361 & 0.495 & 0.141 & 0.493 & 0.04941 \\
\hline 10 & 0.341 & 0.651 & 0.13 & 0.0566 & 0.204 \\
\hline 11 & 0.375 & 0.58 & 0.195 & 0.403 & 0.04892 \\
\hline 12 & 0.444 & 0.579 & 0.005042 & 0.09468 & 0.04537 \\
\hline 13 & 0.725 & 0.001417 & 0.392 & 0.109 & 0.08649 \\
\hline 14 & 0.71 & 0.01602 & 0.416 & 0.07683 & 0.105 \\
\hline 15 & 0.751 & 0.02287 & 0.409 & 0.01455 & 0.04841 \\
\hline 16 & 0.701 & 0.02581 & 0.488 & 0.007717 & 0.03695 \\
\hline 17 & 0.69 & 0.431 & 0.21 & 0.173 & 0.04255 \\
\hline 18 & 0.702 & 0.294 & 0.201 & 0.163 & 0.0888 \\
\hline 19 & 0.697 & 0.03729 & 0.23 & 0.226 & 0.186 \\
\hline 20 & 0.507 & 0.113 & 0.236 & 0.193 & 0.05132 \\
\hline 21 & 0.464 & 0.06074 & 0.424 & 0.366 & 0.115 \\
\hline 22 & 0.647 & 0.297 & 0.03303 & 0.01923 & 0.258 \\
\hline 23 & 0.675 & 0.207 & 0.03394 & 0.122 & 0.03222 \\
\hline 24 & 0.731 & 0.001906 & 0.148 & 0.181 & 0.251 \\
\hline 25 & 0.63 & 0.219 & 0.137 & 0.108 & 0.372 \\
\hline 26 & 0.723 & 0.04318 & 0.04147 & 0.001091 & 0.242 \\
\hline 27 & 0.679 & 0.156 & 0.0301 & 0.24 & 0.0855 \\
\hline 28 & 0.697 & 0.137 & 0.0008682 & 0.116 & 0.07312 \\
\hline 29 & 0.676 & 0.377 & 0.08189 & 0.09546 & 0.24 \\
\hline 30 & 0.666 & 0.389 & 0.02434 & 0.317 & 0.105 \\
\hline
\end{tabular}

\section{DiferenciasporSexo}

Como se puede observar en la tabla $\mathrm{N}^{\circ} 8$, exis- la escala total $(\mathrm{p}<0.001)$ entre ambos sexos, favoreten diferencias significativas en las puntuaciones de ciendo al grupo de mujeres. 


\section{Diferencias por nivel deEscolaridad}

Como se puede observar en la tabla $\mathrm{N}^{\circ} 9$, no existen diferencias significativas en los puntajes de la escala total entre los distintos grupos de escolaridad. Esto indica que no sería necesario establecer Normas según edad de los niños en el primer ciclo básico.

\section{Tabla N $^{\circ} 8$}

\section{Comparación de Puntajes Totales según Sexo.}

\begin{tabular}{lccccc}
\hline Sexo & $N$ & Media & $\begin{array}{c}\text { Desviación } \\
\text { Estandar }\end{array}$ & Mínimo & Máximo \\
\hline Femenino & 159 & 64,975 & 14,285 & 27 & 90 \\
Masculino 162 & 59,617 & 15,055 & 14 & 90 \\
Total & 321 & 62,271 & 14,899 & 14 & 90 \\
& & $\mathbf{F}=\mathbf{1 0 , 6 9}$ & $\mathbf{p}<0,001$ & & \\
\end{tabular}

\begin{tabular}{|cccccc|}
\hline \multicolumn{6}{|c|}{ Tabla N $^{\circ} \mathbf{9}$} \\
Comparación de Puntajes Totales \\
según Escolaridad. \\
\hline Curso & N & Media & Desviac & Mínimo & Máximo \\
/Estand. & & \\
\hline 1 & 93 & 59,473 & 13,264 & 27 & 90 \\
2 & 80 & 62,663 & 16,741 & 14 & 88 \\
3 & 72 & 63,236 & 13,374 & 31 & 86 \\
4 & 76 & 64,368 & 15,863 & 28 & 90 \\
Total & 321 & 62,271 & 14,899 & 14 & 90 \\
\multicolumn{5}{|c|}{$\mathbf{F = 1 , 7 2 6}$} & $\mathbf{p}<\mathbf{0 , 1 6 2}$ \\
\end{tabular}

\section{Conclusiones y proyecciones}

A partir de los resultados estadísticos obtenidos, se puede concluir que la Escala para evaluar la Inteligencia Emocional es altamente confiable, tanto en la prueba total como en cada una de sus dimensiones. Sin embargo, los resultados del análisis factorial motivan la realización de nuevos estudios que permitan esclarecer la configuración del constructo de Inteligencia Emocional, en cuanto a la existencia de sus cinco dimensiones, dado que en esta investigación, no se confirma la presencia de dimensiones claramente delimitadas.

Al mismo tiempo, es recomendable la realización de estudios adicionales que apunten a observar la probable correlación entre la presente Escala y otros instrumentos que evalúen el desarrollo socioemocional infantil en sus distintas áreas. Esto con el objeto de precisar el nivel de autonomía y distintividad del concepto de Inteligencia Emocional, con respecto a otros constructos que se han utilizado tradicionalmente para describir y evaluar el funcionamiento psicológico del niño.

Por otra parte, los análisis complementarios permitieron establecer diferencias significativas según sexo y nivel socioeconómico, lo que apoya la necesidad de construir normas para dichos grupos .

Actualmente, la Escala se encuentra en la última fase del proceso de estandarización en lo que respecta a la Escala Total. No se realizará una estandarización por subescalas como consecuencia de los resultados del análisis factorial antes mencionado.

La Escala de Evaluación de la Inteligencia Emocional (EEIE) es un instrumento de fácil y rápida aplicación, que permitirá a los profesores contar con una herramienta adicional para conocery evaluar el área emocional y social de sus alumnos, detectar posibles factores de riesgo en elárea de la salud mental e implementar consecuentemente estrategias de intervención adecuadas a cada grupo en particular. Esto se torna hoy especialmente relevante, considerando que los Objetivos Transversales de la ReformaEducacional ponen su acento en el desarrollo de destrezas sociales y emocionales en los niños.

\section{Referencias}

Cifuentes, O.; Frene, A.; Sanhueza,P. y Valenzuela, P. «Estandarizacion de la Escala de Evaluacion de la Inteligencia Emocional Escolar» (1999). Tesis de Grado. Concepción: U. San Sebastián, Esc. Psicología.

Gardner, H. (1987). Estructuras de la Mente. México: Fondo de Cultura Económica.

Goleman, D. (1996). La Inteligencia Emocional. Buenos Aires: Vergara. 
Goleman, D. (1998). Working with Emotional Intelligence. New York: Bantam Books.

Henníquez, Y., Saez, M., Valenzuela, C. y Villena, R. (1998). Construcción de una Escala Piloto de Evaluación de la Inteligencia Emocional para Niños de Primer Ciclo Básico. Seminario de Titulación. Concepción: U. San Sebastián, Esc. Psicologia.

Molero, C. y Saiz, E. (1998). "Revisión Histórica del Concepto de Inteligencia: Una Aproximación a la Inteligencia Emocional”. Revista Latinoamericana de Psicología, Vol30 (1), 11-30.
Shapiro, L. (1997). La Inteligencia Emocional en los Niños. Buenos Aires: Vergara.

Young, Ch. (1998). Validity Issues in Measuring Psychological Constructs- The Case of

Emotional Intelligence. http://Trochim. cornell.edu/tutorial/ young/eiweb2.htm.

Young, Ch. (1998). Emotions and Emotional Intelligence. http:/ /trochim.human.cornell.edu/gallery/young/emotion.htm. 